\title{
Variance-Based Robust Optimization of a Permanent Magnet Synchronous Machine
}

\author{
Piotr A. Putek ${ }^{1}$, E. Jan W. ter Maten ${ }^{1}$, Michael Günther ${ }^{1}$, and Jan K. Sykulski ${ }^{2}$, Fellow, IEEE \\ ${ }^{1}$ Chair of Applied Mathematics and Numerical Analysis, Bergische Universität Wuppertal, 42119 Wuppertal, Germany \\ ${ }^{2}$ Electronics and Computer Science, University of Southampton, Southampton SO17 1BJ, U.K.
}

This paper focuses on the application of the variance-based global sensitivity analysis for a topological derivative method in order to solve a stochastic nonlinear time-dependent magnetoquasi-static interface problem. To illustrate the approach a permanent magnet (PM) synchronous machine has been considered. Our key objective is to provide a robust design of the rotor poles and of the tooth base in a stator for the reduction of the torque ripple and electromagnetic losses, while taking material uncertainties into account. Input variations of material parameters are modeled using the polynomial chaos expansion technique, which is incorporated into the stochastic collocation method in order to provide a response surface model. Additionally, we can benefit from the variancebased sensitivity analysis. This allows us to reduce the dimensionality of the stochastic optimization problems, described by the random-dependent cost functional. Finally, to validate our approach, we provide the 2-D simulations and analysis, which confirm the usefulness of the proposed method and yield a novel topology of a PM synchronous machine.

Index Terms - Chaos polynomials, design optimization, permanent magnet (PM) motors, robustness, stochastic processes, topological derivative, uncertainty quantification (UQ).

\section{INTRODUCTION}

D UE to the several attractive features, such as high efficiency and power factor, high torque to weight ratio and brushless construction, permanent magnet (PM) synchronous machines have found recently a wide range of applications in the automotive industry [1]. However, in spite of their unquestionable advantages, including also the field weakening capability of $1: 5$, the electrically controlled PM excited synchronous machine (ECPSM) [2], served here as a case study, suffers inherently from the considerable level of the torque pulsation [3]. This, in turn, may result in the mechanical vibration and acoustic noise and, as a consequence, affect the machine performance. From this perspective, the mitigation of the torque fluctuations is a key issue for the design of a PM machine.

Yet, in many engineering applications, physical models are very often affected by a relatively large amount of uncertainty, which is caused mainly by the imperfections in manufacturing processes. For example, as a result of the punching and quenching in the assembly of the electric machine, some magnetic properties of the core material can deteriorate [4]. Thus, the solution to the optimization problem may be strongly affected by the uncertainties in both the geometrical and material parameters [5]. In the literature, various methods for suppressing the torque ripple (TR) have been proposed. In general, they are divided into two main groups of the deterministic and stochastic optimization methods. Within this context, the topology optimization in particular seems to be a very powerful methodology [3], [6], [7]. Other approaches, which aim to reduce the performance variability, are based on

Manuscript received June 27, 2017; revised August 8, 2017; accepted August 25, 2017. Corresponding author: P. A. Putek (e-mail: putek@ math.uni-wuppertal.de).

Color versions of one or more of the figures in this paper are available online at http://ieeexplore.iee.org.

Digital Object Identifier 10.1109/TMAG.2017.2750485 the statistically originated Taguchi method [8], [9]. Different efficient solutions involve, for example, the use of a gradient indexed method or a perturbation method in order to deal with the "deterministic" variations of some model parameters [10].

From this perspective, there is a need to include uncertainty quantification (UQ) in the modeling phase to obtain reliable numerical simulations. For this reason, in this paper, we explore the stochastic collocation method (SCM) combined with the polynomial chaos expansion [11]. This allows for constructing a suitable surrogate-the so-called response surface model-which can be further incorporated into the topology optimization flow [12], [13]. Our new contribution, in comparison with [13], is first to attain the low TR design of the ECPSM under uncertainties in the transient analysis. Second, the methodology for solving a stochastic timedependent magnetoquasi-static interface problem is based on the variance-based sensitivity analysis. The low TR design with decreased energy consumption profile, found in this way, allows for improving the PM machine quality by minimizing variations of the output performance functions.

\section{StOchastic Forward Problem}

Specifically, for the optimization, when using gradient methods, it is required to consider an efficient computational model. In this respect, the 3-D forward problem has been simplified into the 2-D FE model, which still yields accurate computational results [13]. Thus, a 2-D model can be described using the magnetic vector potential $A$ for the stochastic quasi-linear system of PDEs, defined on $t \in(0, T]$ with $T>0$ and $\mathbf{x}=(x, y)^{\top} \in \mathrm{D} \subset \mathbb{R}^{2}$ as

$$
\left\{\begin{array}{l}
\nabla \cdot\left(v_{\mathrm{Fe}}\left(\mathbf{x},|\nabla A(\theta)|^{2}, \xi_{1}\right) \nabla A(\theta)\right)+\sigma\left(\xi_{4}\right) \partial_{t} A(\theta)=J_{i}(\mathbf{x}, t) \\
\nabla \cdot\left(v_{\mathrm{air}}\left(\mathbf{x}, \xi_{2}\right) \nabla A(\theta)\right)=0 \\
\nabla \cdot\left(v_{\mathrm{PM}}\left(\mathbf{x}, \xi_{3}\right) \nabla A(\theta)\right)=\nabla \cdot v_{\mathrm{PM}}\left(\mathbf{x}, \xi_{3}\right) \mathbf{M}(\mathbf{x})
\end{array}\right.
$$

0018-9464 (C) 2017 IEEE. Personal use is permitted, but republication/redistribution requires IEEE permission.

See http://www.ieee.org/publications_standards/publications/rights/index.html for more information. 
endowed with both boundary and initial conditions, where $\theta:=(\mathbf{x}, t ; \xi) \in D \times(0, T] \times \Omega$ with the domain $\mathrm{D}$, which refers to the sextant region; $\sigma\left(\xi_{4}\right)=\sigma_{\mathrm{Fe}}\left(1+\delta_{4} \xi_{4}\right)$ represents conductivity. $J_{i}(\mathbf{x}, t), i=1,2,3$ denotes an excitation current density and $\Omega$ is a sample space; $\mathbf{M}$ represents the remanent flux density of the PM, while $\boldsymbol{v}$ denotes the reluctivity. In particular, a stochastic model for $v(\cdot ; \xi)$ is given by

$$
v(\theta)= \begin{cases}v_{\mathrm{Fe}}\left(\mathbf{x},|\nabla A(\theta)|^{2}\right)\left(1+\delta_{1} \xi_{1}\right) & \text { for } \mathbf{x} \in D_{\mathrm{Fe}} \\ v_{\mathrm{air}}(\mathbf{x})\left(1+\delta_{2} \xi_{2}\right) & \text { for } \mathbf{x} \in D_{\mathrm{air}} \\ v_{\mathrm{PM}}(\mathbf{x})\left(1+\delta_{3} \xi_{3}\right) & \text { for } \mathbf{x} \in D_{\mathrm{PM}}\end{cases}
$$

where $\xi=\left(\xi_{1}, \xi_{2}, \xi_{3}, \xi_{4}\right)$ are assumed to be random variables, defined on some probability space $(\Omega, \mathcal{F}, \mathbb{P})$ with the event space $\Omega$, sigma-algebra $\mathcal{F}$, and probability measure $\mathbb{P}$. For the scalings $\delta_{j}$, see the following.

\section{UNCERTAINTY QUANTIFICATION AND SOBOL DECOMPOSITION}

For the UQ, we consider $\mathbf{p}(\boldsymbol{\xi})=\left[v_{\mathrm{Fe}}\left(\xi_{1}\right), v_{\mathrm{air}-\mathrm{gap}}\left(\xi_{2}\right)\right.$, $\left.v_{\mathrm{PM}}\left(\xi_{3}\right), \sigma\left(\xi_{4}\right)\right] \in \Pi$, where $\xi_{j}, j=1, \ldots, 4$ are independent and identically uniformly distributed in the interval $[-1,1]^{4}$ with the constant magnitude $\delta_{j}=0.1$, for $j=1,2,3$ and $\delta_{4}=0.05$. Thus, we assume a joint probability density function $g: \Pi \rightarrow \mathbb{R}$, with $\mathbb{P}$, that defines an inner-product $\langle., .\rangle_{g}$ and a space of $L_{g}^{2}$ quadratically integrable functions $u$ that can be approximated by

$$
u(\mathbf{x}, t ; \mathbf{p}) \doteq \sum_{i=0}^{N} \alpha_{i}(\mathbf{x}, t) \Psi_{i}(\mathbf{p})
$$

with a priori unknown coefficient functions $\alpha_{i}$ and predetermined basis polynomials $\Psi_{i}$ with the orthogonality property $\mathbb{E}\left[\Psi_{i} \Psi_{j}\right]=\delta_{i j}$. Here, $\mathbb{E}$ is the expected value, associated with $\mathbb{P}$. For the calculation of $\alpha_{i}$, we applied the SCM with the Stroud-3 formula [13], which yields the solution at each quadrature node $\xi^{(k)}, k=1, \ldots, K$ of the problem (1). Next, the multi-dimensional quadrature rule with associated weights $w_{k}$ is used for projecting function $u_{k}$ into the basis $\Psi_{i}$

$$
\alpha_{i}(\mathbf{x}, t) \doteq \sum_{k=1}^{K} u\left(\mathbf{x}, t, \mathbf{p}^{(k)}\right) \Psi_{i}\left(\mathbf{p}^{(k)}\right) w_{k}
$$

Finally, the statistical moments are approximated by

$$
\mathbb{E}[u(\mathbf{x}, t ; \mathbf{p})] \doteq \alpha_{0}(\mathbf{x}, t), \quad \operatorname{Var}[u(\mathbf{x}, t ; \mathbf{p})] \doteq \sum_{i=1}^{N}\left|\alpha_{i}(t)\right|^{2}
$$

assuming $\Psi_{0}=1$ [11]. Additionally, in order to assess the impact of each uncertain parameter on the output variation, the variance-based sensitivity analysis has been applied. It is based on the Sobol indices, which allow for decomposing the total variance in the form [14]

$$
S:=\sum_{k=1}^{K} S_{i}+\sum_{1 \leq i,<j, \leq K} S_{i j}+\cdots+S_{1,2, \ldots, K}
$$

Here, $S=\operatorname{Var}[u(\mathbf{x}, t ; \mathbf{p})]$ and $S_{i_{1} \ldots, i_{S}}$ denote the total and partial variances, while the PC-based Sobol indices are defined using (3) as [15]

$$
\mathrm{SU}_{i_{1}, \ldots, i_{S}}:=S_{i_{1}, \ldots, i_{S}} / S
$$

where

$$
S \doteq \sum_{|k|=1}^{K} v_{k}^{2}, \quad S_{i_{1} \ldots, i_{S}} \doteq \sum_{|k| \leq K, k \in L} v_{k}^{2}
$$

with $L:=\left\{k \mid k_{i} \geq 1, i \in\left\{i_{1}, \ldots, i_{S}\right\} ; k_{j}=0, j \notin\left\{i_{1}, \ldots, i_{S}\right\}\right\}$. Moreover, the total sensitivity indices can be easily computed, when mixed terms are involved in the summation, see [15].

\section{Stochastic Optimization Problem}

For the optimization, it is necessary to define some criteria, which allow to assess the design of a PM machine. On the one hand, our objective is to suppress the TR. Therefore, as the first objective, we consider the electromagnetic torque (ET) $T$, which in practical applications can be calculated using the virtual work method for a specific position as a partial derivative of the magnetic co-energy $W_{M}$ with respect to the angular displacement as $\vartheta[1]$

$$
T(\theta):=\frac{\partial W_{M}}{\partial \vartheta} .
$$

It should be noted that the ET fluctuations are proportional to the partial derivative of the co-energy stored in a system versus time $\partial_{t} W_{M}$. On the other hand, our second objective is to minimize electromagnetic losses, defined as

$$
P_{\mathrm{avg}}(\theta):=\int_{D} Q(\theta) \mathrm{d} \mathbf{x}+\frac{1}{2} \int_{D} \partial_{t} W_{M}(\theta) \mathrm{d} \mathbf{x}
$$

where the first term of the above-mentioned equation refers to the Joule loss, while the second one-in the case of the time periodic excitation-can be interpreted as the energy dissipation due to the irreversible material behavior (hysteresis). Therefore, we formulate the time-dependent stochastic magnetoquasi-static interface problem for the cost functional, which allows for considering both objectives, by incorporating (9) into (10)

$$
F(\xi):=\frac{1}{2}\left[\int_{0}^{T}\left|P_{r}\left(\partial_{t} A(\mathbf{x}, \boldsymbol{\xi})\right)\right|^{2}+\left|P_{h}\left(\partial_{t} \nabla A(\mathbf{x}, \boldsymbol{\xi})\right)\right|^{2}\right] \mathrm{d} t
$$

with the first term $P_{r}\left(\partial_{t} A(\mathbf{x}, \boldsymbol{\xi})\right):=\int_{D} \sigma \partial_{t} A \cdot \partial_{t} A \mathrm{~d} \mathbf{x}$ and $P_{h}\left(\partial_{t} \nabla A(\mathbf{x}, \boldsymbol{\xi})\right):=\int_{D} \partial_{t} v \nabla A \cdot \nabla A \mathrm{~d} \mathbf{x}$. Here, we just multiply the hysteresis losses by a factor of two, instead of using the average weighted method. Finally, our stochastic optimization problem with the discreet weak form of (1) as the stochastic constraint has been defined as

$$
\begin{array}{ll}
\underset{\mathbf{p}}{\min } & \mathbb{E}[F(\mathbf{p})] \\
\text { s.t.: } & \mathbf{K}\left(\mathbf{p}^{k}\right) \mathbf{A}^{k}=\mathbf{f}^{k}, \quad k=1, \ldots, K \\
& g_{1}(\mathbf{x})=\left|D_{\mathrm{FEr}}\right| /\left|D_{\mathrm{FEr} 0}\right|-S_{\mathrm{FEr}}=0 \\
& g_{2}(\mathbf{x})=\left|D_{\mathrm{PM}}\right| /\left|D_{\mathrm{PM} 0}\right|-S_{\mathrm{PM}}=0 \\
& g_{3}(\mathbf{x})=\left|D_{\mathrm{FEs}}\right| /\left|D_{\mathrm{FEs} 0}\right|-S_{\mathrm{FEs}}=0
\end{array}
$$

where $g_{1}(\mathbf{x}), g_{2}(\mathbf{x})$, and $g_{3}(\mathbf{x})$ are the deterministic area constraints $|D|$ related to the initial areas of iron $\left(D_{\mathrm{FEr} 0}\right)$ and PM, $\left(D_{\mathrm{PM} 0}\right)$ rotor poles and the tooth base in the stator $\left(D_{\mathrm{FEs} 0}\right)$, with some prescribed coefficients, such as $S_{\mathrm{FEr}}, S_{\mathrm{PM}}$, and $S_{\text {FEs }}$, respectively. The stiffness and mass matrix is denoted by $\mathbf{K}$. Furthermore, in order to solve the above-mentioned optimization problem, we can benefit from the Sobol decomposition and use the partial derivative of the variances (7) or the 
TABLE I

Main Parameters of the ECPSM Design [3]

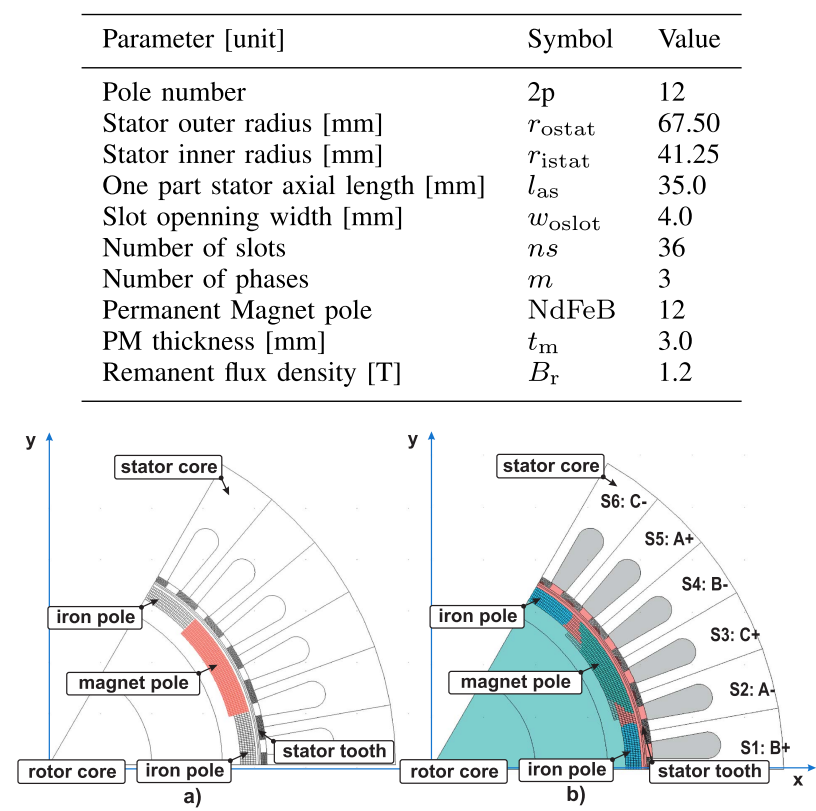

Fig. 1. ECPSM topology for (a) initial and (b) optimized configurations.

partial derivative of the variances with terms of the mixed indices included. In this way, it is also possible to reduce the dimensionality of the optimization problem by eliminating those variables that contribute lowest to the variance-based sensitivity.

\section{Numerical Results AND Discussion}

We applied the proposed procedure to design both the rotor poles as well as the base tooth in the stator of the ECPSM machine at on-load state. The main parameters of the PM are given in Table I. For the simulation of a 2-D finite elements method (FEM) model at the on-load condition, COMSOL 3.5a has been applied with $I_{i}(\mathbf{x}, t)=15[A], i=1,2,3$. We implemented the variance-based algorithm for the topology optimization using MATLAB scripts in MATLAB 7.10. The FEM model consists of a triangular mesh with the second order Lagrange polynomials. The areas of the one-pole pair rotor in the initial 2-D model were divided into 360 and 480 voxels for the iron and the PM poles, respectively. In addition, the base tooth in the stator consisted of 512 voxels. As a reference model, we used the model shown in Fig. 2(a) with the same area of the PM rotor pole as in the optimized structure, shown on Fig. 1(b). Hence, we investigated only the impact of the shape change of the PM rotor poles, including shape changes of iron poles and tooth base in the stator, on the performance functions. To control the geometrical complexity of the various shapes, a version of the level set method based on the topological derivative as introduced in [16] can be used together with the regularization term, as in [7], [13], and [17]. Moreover, to solve a stochastic direct problem, represented by (1), the SCM with the PC expansion has been applied, where the input variations have been described by a uniform distribution. Due to the assumed probabilistic density function, the Legendre polynomials up to order 2 have been used as the basis. Moreover, the application of the Stroud-3 formula for the ECPSM machine with four random parameters leads to
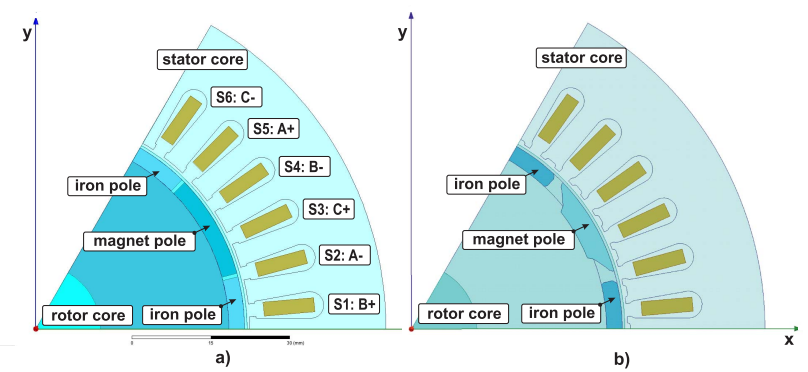

Fig. 2. ECPSM topology for (a) reference and (b) optimized configurations.

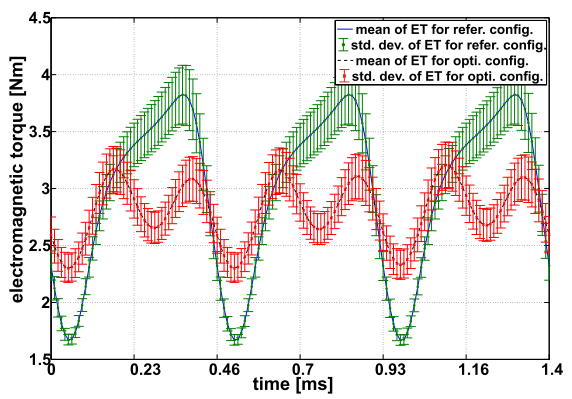

Fig. 3. Mean and standard deviation of the ET for reference and optimized ECPSM configurations.

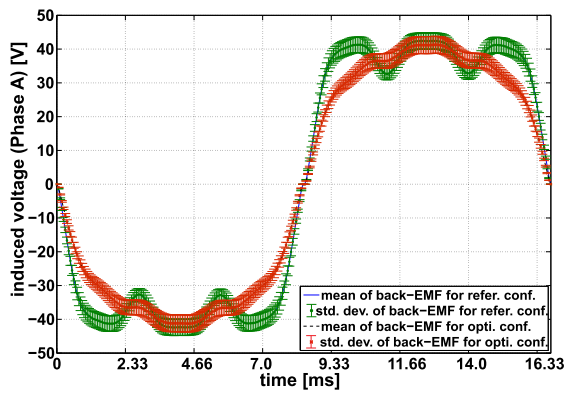

Fig. 4. Mean and standard deviation of the back EMF for reference and optimized ECPSM configurations.

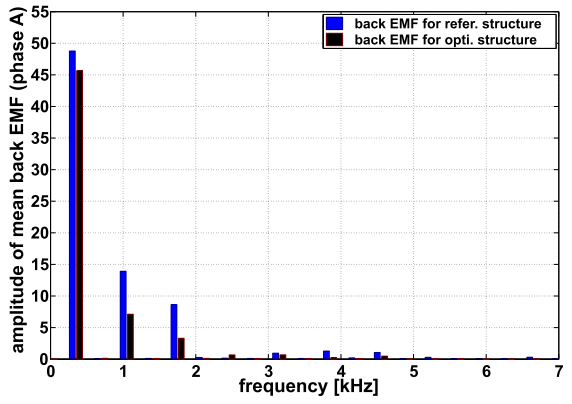

Fig. 5. FFT analysis of the back EMF for reference and optimized ECPSM configurations.

$K=8$ grid points in the 4-D parameter space. The structure of the electric machine, with the optimized shapes of rotor poles and stator teeth, has been found in the 12th iteration of the optimization process, after approximately $8 \mathrm{~h}$ of computing. Both structures, before and after the optimization, are shown in Figs. 1 and 2, respectively. Subsequently, for reference and optimized topology, the ET is calculated over three cycles. The results for the mean and standard deviations of the ET are shown in Fig. 3. In order to investigate the influence of the robust optimization on the back electromotive force (EMF), we present the mean and standard deviations of the back EMF in Fig. 4. In addition, the spectral analysis applying fast Fourier transform (FFT) to the back EMF, as shown in Fig. 5, has been carried out. 


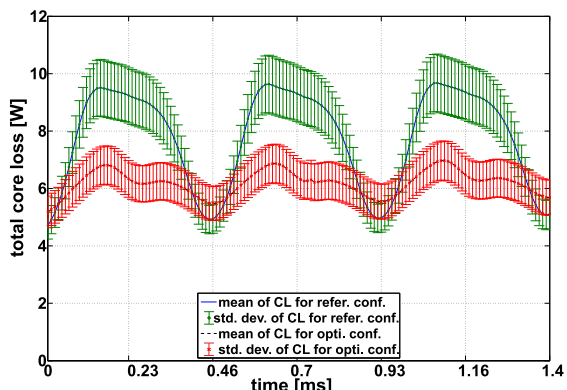

Fig. 6. Mean and standard deviation of the CL for reference and optimized ECPSM configurations.

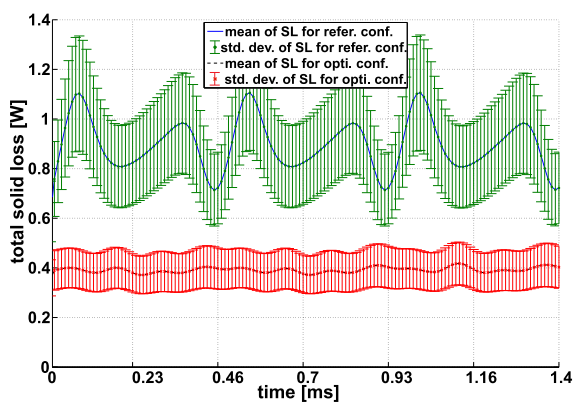

Fig. 7. Mean and standard deviation of the SL (resistive loss) for reference and optimized ECPSM configurations.

TABLE II

Statistics OF SOME PhysicAl PARAMETERS OF THE ECPSM REFERENCE AND Optimized Models at On-LoAd Mode

\begin{tabular}{|c|c|c|c|}
\hline Quantity [unit] & $\begin{array}{c}\text { Reference } \\
\text { topology }\end{array}$ & $\begin{array}{l}\text { Optimized } \\
\text { topology }\end{array}$ & $\begin{array}{l}\text { Decrease } \\
\text { /increase }\end{array}$ \\
\hline \multicolumn{4}{|c|}{ Expectation of the ET [Nm] } \\
\hline Rectified mean value & 2.986 & 2.805 & $6.05 \% \downarrow$ \\
\hline RMS value & 3.067 & 2.818 & $8.12 \% \downarrow$ \\
\hline \multicolumn{4}{|c|}{ Std. dev. of the ET [Nm] } \\
\hline Rectified mean value & 0.166 & 0.165 & $0.71 \% \downarrow$ \\
\hline RMS value & 0.177 & 0.166 & $6.25 \% \downarrow$ \\
\hline \multicolumn{4}{|c|}{ Expectation of the FL [Wb] } \\
\hline Rectified mean value & 0.014 & 0.013 & $5.46 \% \downarrow$ \\
\hline RMS value & 0.016 & 0.015 & $6.65 \% \downarrow$ \\
\hline \multicolumn{4}{|c|}{ Std. dev. of the FL [mWb] } \\
\hline Rectified mean value & 0.751 & 0.750 & $0.15 \% \downarrow$ \\
\hline RMS value & 0.862 & 0.844 & $2.13 \% \downarrow$ \\
\hline \multicolumn{4}{|c|}{ Expectation of the CL $[\mathrm{W}]$} \\
\hline Rectified mean value & 7.812 & 6.198 & $20.66 \% \downarrow$ \\
\hline RMS value & 7.984 & 6.212 & $22.19 \% \downarrow$ \\
\hline \multicolumn{4}{|c|}{ Std. dev. of the CL [W] } \\
\hline Rectified mean value & 0.808 & 0.638 & $21.06 \% \downarrow$ \\
\hline RMS value & 0.827 & 0.638 & $22.85 \% \downarrow$ \\
\hline \multicolumn{4}{|c|}{ Expectation of the SL [W] } \\
\hline Rectified mean value & 0.896 & 0.392 & $56.27 \% \downarrow$ \\
\hline RMS value & 0.902 & 0.391 & $56.54 \% \downarrow$ \\
\hline \multicolumn{4}{|c|}{ Std. dev. of the SL [W] } \\
\hline Rectified mean value & 0.185 & 0.080 & $56.51 \% \downarrow$ \\
\hline RMS value & 0.186 & 0.080 & $56.80 \% \downarrow$ \\
\hline \multicolumn{4}{|c|}{ Mean of others quantities } \\
\hline Ripple torque [\%] & 70.31 & 32.13 & $54.30 \% \downarrow$ \\
\hline
\end{tabular}

In practical calculations for the estimation of the electromagnetic losses, the Berttoti model has been applied [18]. The results of this analysis for both configurations have been shown in Figs. 6 and 7, respectively. Finally, the results for the topology optimization for the quantities, such as the ET, the flux linkage, the core loss (CL), and the solid loss (SL), have been summarized in Table II.

\section{CONCLUSION}

The variance-based topology optimization has been successfully implemented to achieve a low TR as well as reduce electromagnetic losses using a 2-D design of an ECPSM. The proposed procedure results in suppressing the mean rms value of the ripple torque by $54 \%$, and in reducing the mean rms value of the CL and the SL by $22 \%$ and $56 \%$, respectively. A drawback of the proposed method is the associated reduction of the rms value of the ET by $8 \%$, and this issue will be addressed in the future research.

\section{REFERENCES}

[1] F. Gieras and M. Wing, Permanent Magnet Motor Technology. Hoboken, NJ, USA: Wiley, 2008.

[2] H. May, R. Palka, P. Paplicki, S. Szkolny, and W.-R. Canders, "New concept of permanent magnet excited synchronous machines with improved high-speed features," Arch. Elect. Eng., vol. 60, no. 4, pp. 531-540, 2011.

[3] P. Putek, P. Paplicki, and R. Palka, "Low cogging torque design of permanent magnet machine using modified multi-level set method with total variation regularization," IEEE Trans. Magn., vol. 50, no. 2, pp. 657-660, Feb. 2014.

[4] Z. Gmyrek, A. Cavagnino, and L. Ferraris, "Estimation of the magnetic properties of the damaged area resulting from the punching process: Experimental research and FEM modeling," IEEE Trans. Ind. Appl., vol. 49, no. 5, pp. 2069-2077, Sep./Oct. 2013.

[5] P. Sergeant, G. Crevecoeur, L. Dupré, and A. van den Bossche, "Characterization and optimization of a permanent magnet synchronous machine," COMPEL-Int. J. Comput. Math. Elect. Electron. Eng., vol. 28, no. 2, pp. 272-284, 2008.

[6] D.-H. Kim, J. K. Sykulski, and D. A. Lowther, "The implications of the use of composite materials in electromagnetic device topology and shape optimization," IEEE Trans. Magn., vol. 45, no. 3, pp. 1154-1156, Mar. 2009.

[7] S. H. Lim, S. J. Min, and J.-P. Hong, "Low torque ripple rotor design of the interior permanent magnet motor using the multi-phase level-set and phase-field concept," IEEE Trans. Magn., vol. 48, no. 2, pp. 907-910, Feb. 2012.

[8] M. S. Islam, R. Islam, T. Sebastian, A. Chandy, and S. A. Ozsoylu, "Cogging torque minimization in PM motors using robust design approach," IEEE Trans. Ind. Appl., vol. 47, no. 4, pp. 1661-1669, Jul./Aug. 2011.

[9] J. T. Li, Z. J. Liu, M. A. Jabbar, and X. K. Gao, "Design optimization for cogging torque minimization using response surface methodology," IEEE Trans. Magn., vol. 40, no. 2, pp. 1176-1180, Mar. 2004.

[10] N.-K. Kim, D.-H. Kim, D.-W. Kim, H.-G. Kim, D. A. Lowther, and J. K. Sykulski, "Robust optimization utilizing the second-order design sensitivity information," IEEE Trans. Magn., vol. 46, no. 8, pp. 3117-3120, Aug. 2010.

[11] D. Xiu, "Efficient collocational approach for parametric uncertainty analysis," Commun. Comput. Phys., vol. 2, no. 2, pp. 293-309, Apr. 2007.

[12] P. Putek, P. Meuris, R. Pulch, E. J. W. ter Maten, W. Schoenmaker, and M. Günther, "Uncertainty quantification for robust topology optimization of power transistor devices," IEEE Trans. Magn., vol. 52, no. 3, Mar. 2016, Art. no. 1700104

[13] P. Putek, R. Pulch, A. Bartel, E. J. W. ter Maten, M. Günther, and K. M. Gawrylczyk, "Shape and topology optimization of a permanentmagnet machine under uncertainties," J. Math. Ind., vol. 6, no. 1, p. 11, 2016.

[14] I. M. Sobol, "Sensitivity estimates for nonlinear mathematical models," Math. Model. Comput. Exp., vol. 1, no. 4, pp. 407-413, 1993.

[15] B. Sudret, "Global sensitivity analysis using polynomial chaos expansions," Rel. Eng. Syst. Saf., vol. 93, no. 7, pp. 964-979, Jul. 2008.

[16] S. Amstutz and H. Andrä, "A new algorithm for topology optimization using a level-set method," J. Comput. Phys., vol. 216, no. 2, pp. 573-588, 2006.

[17] P. A. Putek, "Mitigation of the cogging torque and loss minimization in a permanent magnet machine using shape and topology optimization," Eng. Comput., vol. 33, no. 3, pp. 831-854, 2016.

[18] G. Bertotti, "General properties of power losses in soft ferromagnetic materials," IEEE Trans. Magn., vol. MAG-24, no. 1, pp. 621-630, Jan. 1988 . 